\title{
FRECUENCIA DE NEOPLASIAS CANINAS EN LIMA: ESTUDIO RETROSPECTIVO EN EL PERIODO 1995-2006
}

\author{
Frequency of Canine Neoplasias in Lima: Retrospective Study in the Period \\ OF 1995-2006
}

\author{
Luis E. de Vivero C. ${ }^{1}$, Alfonso Chavera C. ${ }^{1,3}$, Rosa Perales C. ${ }^{1}$, \\ Viviana Fernández C. ${ }^{2}$
}

\section{Resumen}

Se evaluaron las fichas correspondientes a la especie canina del Laboratorio de Patología de la Facultad de Medicina Veterinaria de la Universidad Nacional Mayor de San Marcos, Lima, de los años 1995-2006, para evaluar la frecuencia de neoplasias diagnosticadas histopatológicamente. Se consideraron las variables sexo, edad, raza, origen celular y localización. De 4438 fichas en caninos, 1092 casos correspondieron a neoplasias (24.6\%). En los grupos etáreos de 5 a $<9$ y $\geq 9$ años se encontró la mayor frecuencia de neoplasias ( 37.1 y $35.6 \%$, respectivamente). No se encontró diferencias entre sexos, pero los canes de raza Boxer fueron los más afectados (12.1\%). Las neoplasias malignas fueron más frecuentes $(64.9 \%)$ que las benignas, siendo el tumor venéreo transmisible la neoplasia más frecuente dentro los tumores benignos y el adenocarcinoma mamario el más frecuente dentro los tumores malignos. Las neoplasias de origen epitelial (39.5\%) y a nivel de la glándula mamaria (16.7\%) fueron las más frecuentes.

Palabras clave: caninos, neoplasias, histopatología, frecuencia, estadística

\section{Abstract}

Canine histopathological records from de Pathology Laboratory of the Universidad Nacional Mayor de San Marcos, Lima, during the period of 1995-2006 were evaluated for determining the frequency of neoplasias in relation to sex, age, breed, cellular origin, and localization. Out of 4438 canine records, 1092 corresponded to neoplasia (24.6\%). Higher tumoral frequency was found in age groups of 5 to $<9(37.1 \%)$ and $\geq 9(35.6 \%)$ years old. None differences were found due to sex, but the Boxer breed was the most affected (12.1\%). Malignant neoplasias were most frequent (64.9\%) than benign ones, where the

\footnotetext{
${ }^{1}$ Laboratorio de Histología, Embriología y Patología Veterinaria, ${ }^{2}$ Clínica de Animales Menores, Facultad de Medicina Veterinaria, Universidad Nacional Mayor de San Marcos, Lima

${ }^{3}$ E-mail: achavera03@gmail.com
} 
transmissible venereal tumor was the most frequent benign tumor and the mammary adenocarcinoma was the most frequent malign tumor. Skin and subcutaneous tissue $(39.5 \%)$ and mammary gland $(16.7 \%)$ were the most common sites for neoplasias.

Key words: canines, neoplasms, histopathology, frequency, statistic

\section{INTRODUCCIÓN}

La mayoría de estudios locales sobre tumores en animales domésticos han sido discusiones de la recolección de casos, más no de incidencia (Moulton, 1978); sin embargo, la prevalencia de tumores cancerosos en mascotas se viene incrementando. Reportes de Bronson (1982) y Michell (1999) señalan que alrededor del 23 y $16 \%$ de muertes de perros en EEUU y el Reino Unido, respectivamente, se deberían a causa del cáncer.

En el caso del Perú, estudios realizados en base al total de muestras analizadas en el laboratorio de histopatología de la Facultad de Medicina Veterinaria de la Universidad Nacional Mayor de San Marcos se encontraron frecuencias del $17 \%$ para el periodo 1948-1977 (Takano, 1964; Rodríguez, 1978) y de 28-30\% para el periodo 1978-1994 (Castro, 1985; Díaz, 1990; San Martín, 2005).

Si bien se desconocen las cifras exactas acerca de la incidencia de neoplasias en perros, estimaciones conservadoras sugieren que uno de cada 10 perros desarrollará un tumor durante su vida natural (Morris, 2002). El aparente incremento de procesos tumorales en caninos se debe a factores tales como la mayor preocupación de los dueños por saber la causa del problema de su mascota, una mejora en la relación del Médico Veterinario con el dueño de la mascota, mayores posibilidades para la realización de exámenes citológicos e histopatológicos, aumento del interés en el estudio de neoplasias, la presencia de mayor número de patólogos veterinarios (Withrow y MacEwen, 1996), y la mayor expectativa de vida de los animales de compañía (Dorn, 1976).

El presente estudio tuvo como objetivo contribuir al conocimiento de los tipos de neoplasias que presentan los caninos en la zona de Lima; así como determinar la frecuencia de las principales patologías oncológicas que afectan a los caninos, incluyendo la edad, sexo y localización de la neoplasia en el organismo.

\section{Materiales y Métodos}

Se emplearon los archivos de diagnóstico citológico e histopatológico del Laboratorio de Patología de la Facultad de Medicina Veterinaria (FMV) de la Universidad Nacional Mayor de San Marcos (UNMSM), Lima, Perú, del periodo 19952006, así como datos históricos del laboratorio (Cuadro 1).

En la recopilación de datos de los protocolos citológicos e histopatológicos se registraron las variables correspondientes a edad ( $<1,1-<5,5-<9, \geq 9$ años), sexo, raza, tipo de tumor según su origen celular y su localización en el organismo. La clasificación de los tumores, según su origen celular, se hizo en base a las recomendaciones de Moulton (1978).

La frecuencia de ocurrencia de neoplasias se hizo a través de la fórmula de proporciones $\left(\mathrm{F}_{\mathrm{i}}=\mathrm{n}_{\mathrm{i}} / \mathrm{N}\right)$ donde $\mathrm{F}_{\mathrm{i}}$ es la frecuencia para una característica a analizar, $\mathrm{n}_{\mathrm{i}}$ es el número de casos que presenta dicha característica, y $\mathrm{N}$ es el número total de 
Cuadro 1. Frecuencia (\%) de casos neoplásicos en caninos de origen celular en el Laboratorio de Patología de la Facultad de Medicina Veterinaria de la Universidad Nacional Mayor de San Marcos (periodo 1948-1995)

\begin{tabular}{|c|c|c|c|c|c|c|c|c|c|}
\hline \multirow{2}{*}{$\begin{array}{l}\text { Periodo } \\
\text { (año) }\end{array}$} & \multirow[b]{2}{*}{$\begin{array}{l}\text { Total } \\
\text { (n) }\end{array}$} & \multicolumn{7}{|c|}{ Tipo de neoplasia } & \multirow[b]{2}{*}{ Referencia } \\
\hline & & $\begin{array}{l}\text { Epite- } \\
\text { liales }\end{array}$ & Mixto & $\begin{array}{l}\text { Conec- } \\
\text { tivo }\end{array}$ & $\begin{array}{l}\text { Hemato- } \\
\text { poyético }\end{array}$ & $\begin{array}{l}\text { Endo- } \\
\text { telial }\end{array}$ & $\begin{array}{l}\text { Muscu- } \\
\text { lar }\end{array}$ & $\begin{array}{l}\text { Misce- } \\
\text { láneo }\end{array}$ & \\
\hline $48-63$ & 160 & 37.8 & 13.0 & 13.0 & 13.6 & 0.6 & 2.4 & 18.6 & $\begin{array}{l}\text { Takano } \\
(1964)\end{array}$ \\
\hline $64-77$ & 302 & 34.3 & 6.6 & 19.1 & 16.5 & 1.7 & 1.3 & 20.1 & $\begin{array}{l}\text { Rodríguez } \\
\text { (1978) }\end{array}$ \\
\hline $78-83$ & 245 & 39.1 & 5.7 & 17.6 & 6.9 & 8.6 & 1.2 & 20.8 & $\begin{array}{l}\text { Castro } \\
(1985)\end{array}$ \\
\hline $84-89$ & 114 & 34.6 & 0 & 24.7 & 8.2 & 9.1 & 0 & 16.4 & $\begin{array}{c}\text { Díaz } \\
\text { (1990) }\end{array}$ \\
\hline $90-94$ & 73 & 49.3 & 0 & 13.7 & 16.4 & 6.9 & 0 & 13.7 & $\begin{array}{c}\text { San } \\
\text { Martín } \\
(2005)\end{array}$ \\
\hline
\end{tabular}

casos. Además, se determinaron los intervalos de confianza al $95 \%$ para una proporción.

\section{Resultados y Discusión}

Se revisaron 4438 casos de caninos en el periodo bajo estudio, correspondiendo 1092 casos a neoplasias, lo cual representa el $24.6 \%$ de la casuística del laboratorio. Esta proporción de neoplasias fue un 4-6\% inferior a la reportada en ese mismo laboratorio en el periodo 1984-1995 (Díaz, 1990; San Martín, 2005).

El Cuadro 2 muestra la frecuencia de neoplasias por efecto de la edad, sexo, grupo racial y tipo por origen celular. La mayor frecuencia de neoplasias se encontró en los grupos etarios mayores de 5 años de edad sin que haya diferencia estadística entre los grupos etarios de 5 a $<9$ y $\geq 9$ años. Resultados similares han sido reportados en otros países (Cohen, 1974; Moulton, 1978; Withrow y MacEwen, 1996; Bellei et al., 2006). Las frecuencias de neoplasias por efecto del sexo fueron similares, pese a la ocurrencia de neoplasias propias de cada sexo, como las neoplasias de glándulas mamarias en las hembras y de las glándulas perianales y de testículo en los machos. Por otro lado, el tumor venéreo transmisible afecta a ambos sexos y presenta una alta incidencia en el país (Takano, 1964; Rodríguez; 1978; Castro, 1985; Morris, 2002; Mendoza et al., 2010).

Dentro de las razas puras, el Boxer fue la que mostró una mayor frecuencia de presentación de neoplasias (12.1 $\pm 2 \%$ ), pero los canes cruzados de raza indeterminada presentaron la mayor frecuencia $(34.6 \pm 2 \%)$ (Cuadro 2). No obstante, se debe considerar la gran variabilidad de animales por raza en la ciudad de Lima, y que los mestizos se encuentran en mayor cantidad que canes de razas puras. Por otro lado, estudios realizados 
Cuadro 2. Registro de número de casos y frecuencia de neoplasias en caninos en base a registros de diagnósticos citológicos e histopatológicos del Laboratorio de Patología de la Facultad de Medicina Veterinaria de la Universidad Nacional Mayor de San Marcos, Lima (periodo 1995-2006), según edad, sexo, raza del animal y origen celular del tumor

\begin{tabular}{|c|c|c|c|}
\hline Variable & & $\mathrm{N} .^{\circ}$ de observaciones & Porcentaje $\pm \mathrm{IC}^{1}$ \\
\hline \multirow{4}{*}{ Edad (años) $)^{2}$} & $<1$ & 56 & $5.3 \pm 1$ \\
\hline & $1-<5$ & 233 & $22.0 \pm 2$ \\
\hline & $5-<9$ & 393 & $37.1 \pm 3$ \\
\hline & 9 o más & 377 & $35.6 \pm 3$ \\
\hline \multirow{2}{*}{$\mathrm{Sexo}^{3}$} & Hembra & 543 & $50.7 \pm 3$ \\
\hline & Macho & 529 & $49.4 \pm 3$ \\
\hline \multirow{7}{*}{$\mathrm{Raza}^{4}$} & Boxer & 128 & $12.1 \pm 2$ \\
\hline & Cocker Spaniel Inglés & 71 & $6.7 \pm 2$ \\
\hline & Cruzado & 328 & $30.9 \pm 3$ \\
\hline & Labrador Retriever & 51 & $4.8 \pm 1$ \\
\hline & Pastor Alemán & 54 & $5.1 \pm 1$ \\
\hline & Rottweiler & 62 & $5.8 \pm 1$ \\
\hline & Otros & 368 & $34.6 \pm 3$ \\
\hline \multirow{7}{*}{ Origen celular } & Epiteliales & 490 & $44.2 \pm 3$ \\
\hline & Mixto & 34 & $3.1 \pm 1$ \\
\hline & Conectivo & 193 & $17.4 \pm 2$ \\
\hline & Hematopoyéticos & 129 & $11.6 \pm 2$ \\
\hline & Endotelial & 77 & $6.9 \pm 2$ \\
\hline & Muscular & 41 & $3.7 \pm 1$ \\
\hline & Misceláneos & 145 & $13.1 \pm 2$ \\
\hline Total & & 1109 & 100.0 \\
\hline
\end{tabular}

por Cohen et al. (1974) y Moulton (1978) señalan al Bóxer, Cocker Spaniel, Poodle y Pastor Alemán como las razas más afectadas.

Las neoplasias epiteliales fueron las que ocuparon el primer lugar de importancia (44.2 $\pm 3.0 \%$ ), siendo resultados similares a los reportados en la literatura (Cohen et al., 1974; Moulton, 1978; Scott et al., 2002). Asimismo, las frecuencias encontradas para las variables en estudio fueron similares a las reportadas por Takano (1964), Rodríguez (1978), Castro (1985), Díaz (1990) y San Martín (2005) con datos del laboratorio de 
Cuadro 3. Frecuencia de neoplasias caninas benignas en base a registros de diagnóstico citológicos e histopatológicos del Laboratorio de Patología de la Facultad de Medicina Veterinaria de la Universidad Nacional Mayor de San Marcos, Lima (periodo 1995-2006)

\begin{tabular}{lcc}
\hline Neoplasias Benignas & $(\mathrm{n})$ & $(\%)$ \\
\hline Fibroma & 14 & 3.6 \\
Mixoma & 1 & 0.3 \\
Lipoma & 42 & 10.8 \\
Mastocitoma & 48 & 12.3 \\
Histiocitoma & 17 & 4.4 \\
Hemangioma & 29 & 7.5 \\
Leioma & 4 & 1.0 \\
Papiloma & 50 & 12.9 \\
Adenoma & 69 & 17.7 \\
\multicolumn{1}{c}{ de glándula perianal } & 34 & 8.7 \\
\multicolumn{1}{c}{ de glándula mamaria } & 13 & 3.3 \\
$\quad$ otros adenomas & 22 & 5.7 \\
Tricoepitelioma & 20 & 5.1 \\
Hemangiopericitoma & 6 & 1.5 \\
Epuli fibromatoso & 9 & 2.3 \\
Tumor Venéreo Transmisible & 72 & 18.5 \\
Tumor de Sertoli & 6 & 1.5 \\
Tumor de Leydig & 2 & 0.5 \\
\hline Total & 389 & 100 \\
\hline
\end{tabular}

patología de la FMV-UNMSM en periodos previos.

Las neoplasias malignas fueron más frecuentes $(64.9 \pm 3 \%)$ que las benignas $(35.08 \pm 3 \%)$. Dentro de las neoplasias benignas (Cuadro 3) se encontró al tumor venéreo transmisible (TVT) como el tumor benigno más frecuente, como fuera reportado por Castro (1985), Díaz (1990) y Mendoza et al. (2010). El adenocarcinoma mamario, el linfoma maligno y el carcinoma de células escamosas fueron las neoplasias malignas más frecuentes (Cuadro 4), tal y como fuera encontrado en estudios previos (Castro, 1985; Díaz, 1990; San Martín, 2005).

Según la localización de la neoplasia, la mayor frecuencia de neoplasias se encontró a nivel de piel, tejido subcutáneo (429 casos, $39.5 \%$ ) y glándula mamaria (214 casos, 19.7\%). Estas proporciones fueron similares a las reportadas en otros estudios (Mulligan, 1949; Cohen et al., 1974; Rodríguez, 1978; Dobson et al., 2002). 
Cuadro 4. Frecuencia de neoplasias caninas malignas en base a registros de diagnóstico citológicos e histopatológicos del Laboratorio de Patología de la Facultad de Medicina Veterinaria de la Universidad Nacional May or de San Marcos, Lima (periodo 1995-2006)

\begin{tabular}{|c|c|c|}
\hline Neoplasias Malignas & (n) & $(\%)$ \\
\hline Fibrosarcoma & 43 & 6.0 \\
\hline Mixosarcoma & 2 & 0.3 \\
\hline Condrosarcoma & 7 & 1.0 \\
\hline Osteosarcoma & 9 & 1.3 \\
\hline Osteocondrosarcoma & 3 & 0.4 \\
\hline Liposarcoma & 6 & 0.8 \\
\hline Mastocitosarcoma & 1 & 0.1 \\
\hline Hemangiosarcoma & 47 & 6.5 \\
\hline Linfagiosarcoma & 1 & 0.1 \\
\hline Linfoma & 81 & 11.3 \\
\hline Plasmocitoma & 48 & 6.7 \\
\hline Leiomios arcoma & 8 & 1.1 \\
\hline Rabdomiosarcoma & 29 & 4.0 \\
\hline Carcinoma & 170 & 23.6 \\
\hline de célu las basales & 42 & 5.8 \\
\hline de célu las escamosas & 86 & 11.9 \\
\hline Mamario & 13 & 1.8 \\
\hline Hepático & 3 & 0.4 \\
\hline Otros carcinomas & 39 & 5.4 \\
\hline Adenocarcinoma & 181 & 25.1 \\
\hline Mamario & 134 & 18.6 \\
\hline Glándula perianal & 16 & 2.2 \\
\hline Otros adenocarcinomas & 31 & 4.3 \\
\hline Tumor Mamario Mixto Maligno & 34 & 4.7 \\
\hline Melanoma & 36 & 5.0 \\
\hline Disgerminoma & 1 & 0.1 \\
\hline Seminoma & 13 & 1.8 \\
\hline Total & 720 & 100 \\
\hline
\end{tabular}

\section{Conclusiones}

- Las neoplasias de origen epitelial son las más frecuentes en caninos.

- La mayor frecuencia de neoplasias se presenta en caninos a partir de los cinco años de edad.

- Las neoplasias se presentaron con una frecuencia similar en machos y hembras. Asimismo, la raza más afectada fue el Bóxer. 
- Las neoplasias malignas son más frecuentes que las benignas; siendo el tumor venéreo transmisible la neoplasia benigna más frecuente dentro los tumores benignos y el adenocarcinoma mamario el más frecuente dentro los tumores malignos.

\section{Literatura Citada}

1. Bellei MH, da Silva Neves D, Gava A, Padilha P, Pilati C. 2006. Prevalência de neoplasias cutâneas diagnosticadas em caninos no estado de Santa Catarina, Brasil, no período entre 1998 a 2002. Rev Ciên Agrovet 5(1): 73-79.

2. Bronson RT. 1982. Variation in age at death of dogs of different sexes and breeds. Am J Vet Res 43: 2057-2059.

3. Castro J. 1985. Neoplasias en la especie canina. Estudio estadístico de 301 casos entre 1978-1983. Tesis de Médico Veterinario. Lima. Facultad de Medicina Veterinaria, Univ Nacional Mayor de San Marcos. 42 p.

4. Cohen D, Reif JS, Brodey RS, Keiser $H .1974$. Epidemiological analysis of the most prevalent sites and types of canine neoplasia observed in a veterinary hospital. Cancer Res 34: 2859-2868.

5. Díaz M. 1990. Procesos neoplásicos en caninos. Análisis estadístico. Periodo 1984-1989. Tesis de Médico Veterinario. Facultad de Medicina Veterinaria, Univ Nacional Mayor de San Marcos. $26 \mathrm{p}$.

6. Dobson JM, Samuel S, Milstein H, Rogers K, Wood JLN. 2002. Canine neoplasia in the UK: estimates of incidence rates from a population of insured dogs. J Small Anim Pract 43: 240-246.

7. Dorn CR. 1976. Epidemiology of canine and feline tumors. J Am Anim Hosp Assoc 12:307-312.
8. Mendoza N, Chavera A, Falcón N, Perales $R$. 2010. Frecuencia del tumor venéreo transmisible en caninos: Casuística del Laboratorio de Patología veterinaria de la Universidad Nacional Mayor de San Marcos (Periodo 1998-2004). Rev Inv Vet Perú 21: 42-47.

9. Michell AR. 1999. Longevity of British breeds of dogs and its relationship with sex, size, cardiovascular variables and disease. Vet Rec 145: 625-629.

10. Morris J. 2002. Oncología en pequeños animales. Buenos Aires: Inter-Médica. $280 \mathrm{p}$.

11. Moulton JE. 1978. Tumors in domestic animals. $2^{\text {nd }}$ ed. California, USA: University of California Press. 465 p.

12. Mulligan M. 1949. Neoplasm of the dog. $2^{\text {nd }}$ ed. Baltimore, USA: Williams and Wilkins. $420 \mathrm{p}$.

13. Rodríguez J. 1978. Neoplasias en la especie canina: Estudio estadístico periodo 1964-1977. Tesis de Médico Veterinario. Lima: Facultad de Medicina Veterinaria, Univ Nacional Mayor de San Marcos. 35 p.

14. San Martín M. 2005. Neoplasias caninas: Evaluación estadística. Periodo 1990-1994. Tesis de Médico Veterinario. Lima: Facultad de Medicina Veterinaria, Univ Nacional Mayor de San Marcos. $46 \mathrm{p}$.

15. Scott DW, Miller WH, Griffin CE. 2002. Muller \& Kirk. Dermatología en pequeños animales. $6^{\text {a }}$ ed. Buenos Aires: Inter-Médica. $1572 \mathrm{p}$.

16. Takano F. 1964. Estudio retrospectivo de 154 procesos neoplásicos en la especie canina. Tesis de Médico Veterinario. Lima: Facultad de Medicina Veterinaria, Univ Nacional Mayor de San Marcos. $55 \mathrm{p}$.

17. Withrow S, MacEwen SC. 1996. Small animal clinical oncology. $2^{\text {nd }} e d$. Philadelphia, USA: WB Saunders. 846 p. 